\title{
Antipsychotic drugs for psychosis and agitation in dementia: efficacy, safety, and a possible noradrenergic mechanism of action
}

The first patient described by Alzheimer in 1907 had both progressive cognitive deterioration and prominent comorbid signs and symptoms of psychosis and agitation (Alzheimer, 1907, 1987). In this editorial, we use "psychosis" to denote delusions and hallucinations and "agitation" to denote irritability, aggression, pressured motor activity, and active resistance to necessary care. Although advances have been made in the treatment of these non-cognitive symptoms, these psychosis and agitation symptoms continued to be burdensome and costly for dementia patients, caregivers, and society. Among the pharmacologic treatments available for psychosis and agitation, antipsychotic drugs are the drug class most consistently demonstrated effective for psychosis and agitation in dementia (Lyketsos et al., 2006; APA Work Group on Alzheimer's Disease and Other Dementias et al., 2007). These are widely prescribed for these behavioral problems, but their use remains controversial and their mechanism of action unclear.

In this editorial, we attempt to discern from the clinical trial data which non-cognitive symptoms of dementia, if any, are therapeutically responsive to antipsychotics. We argue that despite the descriptor "antipsychotics," (derived from their clear and substantial efficacy for the complex bizarre delusions and auditory hallucinations of psychosis in schizophrenia), the predominant effect of these drugs in dementia is to reduce agitation rather than psychosis. The most common psychosis symptoms in dementia are simple, memory impairmentinfluenced delusions (e.g., delusions of theft) and visual hallucinations. In contrast to agitation, these common psychosis symptoms in dementia are poorly responsive to antipsychotic drugs (Rabinowitz et al., 2007). We propose a model in which the therapeutic effect of antipsychotic drugs for agitation in dementia is a function of their alpha-1 adrenoreceptor (AR) antagonist activity rather than the dopamine receptor-2 antagonist activity, believed to explain efficacy for the psychosis symptoms of schizophrenia.

Although a detailed review of the now large number of randomized controlled trials (RCTs) of antipsychotic drugs for psychosis and agitation in dementia is beyond the scope of this editorial, we will refer to representative studies. In the 1980 s and early 1990s, several "conventional" antipsychotics were evaluated for "behavioral disturbance" in dementia patients. Loxapine, thioridazine, and thiothixene were modestly effective, but pseudoparkinsonism and excessive sedation often limited usefulness (Barnes et al., 1982; Petrie et al., 1982; Finkel et al., 1995). The subsequently introduced atypical antipsychotics with lower incidences of extrapyramidal adverse effects became widely prescribed for behavioral disturbance in dementia, particularly in long-term care facilities (Kamble et al., 2009). Because these drugs were still under patent protection, several pharmaceutical companies saw an opportunity to obtain Food and Drug Administration (FDA) approval for risperidone, olanzapine, quetiapine, and aripiprazole as effective treatments for "psychosis of dementia" (Katz et al., 1999; Street et al., 2000; De Deyn et al., 2004; 2005; Mintzer et al., 2006; Tariot et al., 2006; Katz et al., 2007; Mintzer et al., 2007; Zhong et al., 2007; Streim et al., 2008). For both regulatory and diagnostic reasons, psychosis of dementia was designated as the primary outcome measure in these large multicenter RCTs. Drug effects on agitation were also measured, but as secondary outcome measures. Disappointingly, these RCTs generally failed to demonstrate meaningful efficacy for psychosis of dementia, the primary outcome measure upon which the FDA approval depended. In contrast, these RCTs consistently demonstrated efficacy for agitation symptoms in dementia. However, the secondary outcome measure designation of "agitation" and the nonspecifity of agitation as a disorder entity worked against the FDA approval of atypical antipsychotics for agitation in dementia. Further support for efficacy of the atypical antipsychotic risperidone for psychosis and/or agitation in Alzheimer's disease (AD) is provided by a placebo-controlled study of relapse rate after discontinuing risperidone in treatment responders (Devanand et al., 2012). Discontinuation of 
risperidone was associated with an increased risk of relapse.

A major safety issue that arose from the pharmaceutical industry development of antipsychotics for psychosis of dementia was a modestly increased risk of death in the active antipsychotic drug groups compared with the placebo groups. A paired analysis of 17 short-term RCTs revealed 1.6 times higher mortality among participants receiving antipsychotics than among participants receiving placebos (Schneider et al., 2005). This finding resulted in an FDA black box warning for these medications in dementia patients. The etiology of the increased mortality risk in these shortterm studies remains unclear. Longer studies in community samples have failed to detect increased mortality risk in dementia patients treated with antipsychotics (Raivio et al., 2007; Simoni-Wastila et al., 2009). It is also possible that psychosis and agitation per se increase mortality and longterm institutionalization risks in dementia patients. A recent long-term longitudinal study of 957 patients with $\mathrm{AD}$ found that use of conventional and atypical antipsychotics was not associated with time to nursing home admission or death (Lopez et al., 2013). Rather, the presence of psychiatric symptoms, including psychosis and agitation, increased risk for institutionalization and death after adjusting for exposure to antipsychotics.

Because the published RCTs of antipsychotic drugs for behavioral disturbance in dementia generally do not present results for discrete psychosis and agitation signs and symptoms, it is difficult to dissect drug effects on specific types of psychotic behaviors (e.g., visual vs. auditory hallucinations) or components of agitation. Fortunately, Rabinowitz and colleagues (2007) evaluated the effects of the widely prescribed atypical antipsychotic risperidone on specific behavioral signs and symptoms in nursing home residents with $\mathrm{AD}$ or mixed etiology dementia. They performed a post hoc exploratory analysis of 479 patients who had participated in one of the three 12-week RCTs and who met criteria for psychosis in AD. Notably, patients in these trials who had agitation but not psychosis (the majority of participants) were not included in the analysis. In spite of selecting patients with moderate or severe psychosis symptoms, significant risperidone effects on individual items were limited to agitation symptoms and signs, including verbal and physical aggression pacing and irritability. Delusions of theft and hallucinations (visual twice as common as auditory) were particularly unresponsive to risperidone. A modestly significant $(\mathrm{p}=0.03)$ therapeutic effect of risperidone was detected only when all psychosis symptoms were merged.
Other than the antipsychotics, the only drug effective for agitation in dementia in more than one placebo-controlled trials is the selective serotonin reuptake inhibitor (SSRI) citalopram. Nyth and Gottfries (1990) demonstrated efficacy of citalopram for agitation (and depression) symptoms in patients with $\mathrm{AD}$ but not in those with vascular dementia. In a short-term trial comparing citalopram with the antipsychotic perphenazine and placebo in dementia patients hospitalized for psychosis and/or agitation, citalopram was more effective than placebo (and tended to be more effective than perphenazine) for agitation but not for psychosis (Pollock et al., 2002).

A recently published multi-center placebocontrolled trial of citalopram in $186 \mathrm{AD}$ patients with agitation provides evidence for clinically meaningful efficacy in this population (Porsteinsson et al., 2014). At a citalopram target dose of $30 \mathrm{mg}$ per day, active drug was significantly superior to placebo for the Neurobehavioral Rating Scale - Agitation subscale, the Clinical Global Impression of Change, and caregiver distress scores. That citalopram was not significantly superior to placebo on the Neuropsychiatric Inventory Agitation subscale (which is heavily loaded with aggressive behaviors) raises the possibility that overtly aggressive behaviors may be less responsive to citalopram than are other agitation behaviors. That said, citalopram is now clearly a treatment option for agitation in AD.

If agitation is responsive to citalopram and is the dementia behavioral disturbance most responsive to antipsychotics, then how can this be explained pharmacologically? We hypothesize that excessive brain noradrenergic system responsiveness contributes to the pathophysiology of agitation in $\mathrm{AD}$. The well-known alpha-1 AR antagonist activity of antipsychotics (Roth et al., 2004; Nasrallah, 2008) and the less well-known but clearly demonstrated ability of SSRIs to decrease both locus coeruleus (LC) neuron norepinephrine (NE) biosynthetic capacity (Nestler et al., 1990) and LC neuron activity (West et al., 2009) could explain the ability of these drugs to reduce agitation in dementia. This hypothesis is consistent with the function of the brain noradrenergic system. Novel environmental stimuli and stressful events stimulate LC NE release to limbic and neocortical regions, increasing arousal and alertness and excessive brain noradrenergic activity, contributing to agitation behaviors, such as motor hyperactivity, irritability, and aggression (Berridge and Waterhouse, 2003; Ramos and Arnsten, 2007).

But how can this hypothesis be reconciled with the substantial loss of LC noradrenergic neurons that occurs in $\mathrm{AD}$ ? Results of postmortem brain 
tissue and clinical studies from our laboratory help resolve this paradox (Peskind et al., 1995; Elrod et al., 1997; Szot et al., 2006; 2007; Wang et al., 2009).

In spite of LC neuronal loss in $\mathrm{AD}$ (Tomlinson et al., 1981; Bondareff et al., 1982; Chan-Palay and Asan, 1989), measurements of $\mathrm{NE}$ and its metabolite 3-Methoxy-4-hydroxyphenylglycol (MHPG) in cerebrospinal fluid (CSF) reveal equivalent levels to those of normal older persons and higher levels than those of normal young persons (Raskind et al., 1984; Elrod et al., 1997). When LC NE outflow is stimulated by yohimbine, $\mathrm{AD}$ patients demonstrate robust CSF $\mathrm{NE}$ increases that are equivalent to those of normal older patients and substantially greater than in young patients (Peskind et al., 1995); AD patients, but not normal older or young patients, developed agitation symptoms following yohimbine. Studies in postmortem brain tissue demonstrate compensatory changes in brain noradrenergic systems consistent with these clinical research findings. In $\mathrm{AD}$ (and in dementia with Lewy bodies), surviving LC neurons demonstrate increased NE biosynthetic capacity (Szot et al., 2006) and there is upregulation of postsynaptic alpha-1 AR and beta AR in limbic and neocortical LC neuronal projection areas (Russo-Neustadt et al., 1998; Szot et al., 2007).

Based on these findings, we evaluated clinically available CNS active AR antagonist drugs as potential therapeutic agents for agitation in AD. The beta AR antagonist propranolol was minimally and only transiently effective (Peskind et al., 2005) (note: antipsychotics do not have beta AR antagonist activity). Thus, beta AR upregulation does not appear to contribute substantially to agitation in $\mathrm{AD}$. The alpha-1 $\mathrm{AR}$ antagonist activity common to all antipsychotic drugs and our demonstration of prazosin efficacy for agitation behaviors in post-traumatic stress disorder (Raskind et al., 2007; 2013) led us to evaluate prazosin for agitation in AD. Prazosin produced a robust and sustained decrease in agitation and improvement in global clinical status in $\mathrm{AD}$ patients with moderate to severe agitation (Wang et al., 2009). Notwithstanding its antihypertensive indication, prazosin gradually titrated upward and was well tolerated without hypotension at $2 \mathrm{mg}$ in the morning and $4 \mathrm{mg}$ in the evening. These results are consistent with the hypothesis that the alpha-1 AR antagonist activity of antipsychotics contributes to their ability to reduce agitation in AD. Because prazosin does not produce pseudoparkinsonism or sedation, this inexpensive drug may prove a useful therapeutic alternative to antipsychotics for agitation symptoms in $\mathrm{AD}$ and other later life dementias. A large multicenter RCT of prazosin for agitation in $\mathrm{AD}$ under the auspices of the National Institute on Aging-funded Alzheimer's Disease Cooperative Study is scheduled to begin in late 2014.

\section{Conflict of interest}

None

\section{MURRAY A. RASKIND AND LUCY Y. WANG}

Veterans Affairs Northwest Network (VISN 20) Mental Illness Research, Education and Clinical Center, Mental Health Service, VA Puget Sound Health Care System, and Department of Psychiatry and Behavioral Sciences, University of Washington, Seattle, Washington, USA Email: murray.raskind@va.gov

\section{References}

Alzheimer, A. $(1907,1987)$. About a peculiar disease of the cerebral cortex (translated by L. Jarvik and H. Greenson). Alzheimer's Disease and Associated Disorders, 1, 3-8.

APA Work Group on Alzheimer's Disease and Other Dementias et al. (2007). American Psychiatric Association practice guideline for the treatment of patients with Alzheimer's disease and other dementias. Second edition. American fournal of Psychiatry, 164, 5-56.

Barnes, R., Veith, R., Okimoto, J., Raskind, M. and Gumbrecht, G. (1982). Efficacy of antipsychotic medications in behaviorally disturbed dementia patients. American fournal of Psychiatry, 139, 1170-1174.

Berridge, C. W. and Waterhouse, B. D. (2003). The locus coeruleus-noradrenergic system: modulation of behavioral state and state-dependent cognitive processes. Brain Research Brain Research Review, 42, 33-84.

Bondareff, W., Mountjoy, C. Q. and Roth, M. (1982). Loss of neurons of origin of the adrenergic projection to cerebral cortex (nucleus locus ceruleus) in senile dementia. Neurology, 32, 164-168.

Chan-Palay, V. and Asan, E. (1989). Alterations in catecholamine neurons of the locus coeruleus in senile dementia of the Alzheimer's type and in Parkinson's disease with and without dementia and depression. Fournal of Comparative Neurology, 287, 373-392.

De Deyn, P. P. et al. (2004). Olanzapine versus placebo in the treatment of psychosis with or without associated behavioral disturbances in patients with Alzheimer's disease. International fournal of Geriatric Psychiatry, 19, 115-126.

De Deyn, P. et al. (2005). Aripiprazole for the treatment of psychosis in patients with Alzheimer's disease: a randomized, placebo-controlled study. Fournal of Clinical Psychopharmacoly, 25, 463-467.

Devanand, D. P. et al. (2012). Relapse risk after discontinuation of risperidone in Alzheimer's disease. New England Fournal of Medicine, 367, 1497-1507. 
Elrod, R., Peskind, E. R., DiGiacomo, L., Brodkin, K. I., Veith, R. C. and Raskind, M. A. (1997). Effects of Alzheimer's disease severity on cerebrospinal fluid norepinephrine concentration. American fournal of Psychiatry, 154, 25-30.

Finkel, S. I. et al. (1995). A randomized, placebo-controlled trial of thiothixene in agitated, demented nursing home patients. International fournal of Geriatric Psychiatry, 10, 129-136.

Kamble, P., Chen, H., Sherer, J. T. and Aparasu, R. R. (2009). Use of antipsychotics among elderly nursing home residents with dementia in the US: an analysis of National Survey Data. Drugs \& Aging, 26, 483-492.

Katz, I., de Deyn, P. P., Mintzer, J., Greenspan, A., Zhu, Y. and Brodaty, H. (2007). The efficacy and safety of risperidone in the treatment of psychosis of Alzheimer's disease and mixed dementia: a meta-analysis of 4 placebo-controlled clinical trials. International fournal of Geriatric Psychiatry, 22, 475-484.

Katz, I. R., Jeste, D. V., Mintzer, J. E., Clyde, C., Napolitano, J. and Brecher, M. (1999). Comparison of risperidone and placebo for psychosis and behavioral disturbances associated with dementia: a randomized, double-blind trial. Risperidone Study Group. Fournal of Clinical Psychiatry, 60, 107-115.

Lopez, O. L. et al. (2013). The long-term effects of conventional and atypical antipsychotics in patients with probable Alzheimer's disease. American fournal of Psychiatry, 170, 1051-1058.

Lyketsos, C. G. et al. (2006). Position statement of the American Association for Geriatric Psychiatry regarding principles of care for patients with dementia resulting from Alzheimer's disease. American fournal of Geriatric Psychiatry, 14, 561-572.

Mintzer, J. et al. (2006). Risperidone in the treatment of psychosis of Alzheimer's disease: results from a prospective clinical trial. American fournal of Geriatric Psychiatry, 14, 280-291.

Mintzer, J. E. et al. (2007). Aripiprazole for the treatment of psychoses in institutionalized patients with Alzheimer's dementia: a multicenter, randomized, double-blind, placebo-controlled assessment of three fixed doses. American fournal of Geriatric Psychiatry, 15, 918-931.

Nasrallah, H. A. (2008). Atypical antipsychotic-induced metabolic side effects: insights from receptor-binding profiles. Molecular Psychiatry, 13, 27-35.

Nestler, E. J., McMahon, A., Sabban, E. L., Tallman, J. F. and Duman, R. S. (1990). Chronic antidepressant administration decreases the expression of tyrosine hydroxylase in the rat locus coeruleus. Proceedings of the National Academy of Sciences USA, 87, 7522-7526.

Nyth, A. L. and Gottfries, C. G. (1990). The clinical efficacy of citalopram in treatment of emotional disturbances in dementia disorders. A Nordic multicentre study. British Fournal of Psychiatry, 157, 894-901.

Peskind, E. R. et al. (1995). Effects of Alzheimer's disease and normal aging on cerebrospinal fluid norepinephrine responses to yohimbine and clonidine. Archives of General Psychiatry, 52, 774-782.

Peskind, E. R. et al. (2005). Propranolol for disruptive behaviors in nursing home residents with probable or possible Alzheimer's disease: a placebo-controlled study.
Alzheimer Disease E Associated Disorders, 19, 23-28.

Petrie, W. M. et al. (1982). Loxapine in psychogeriatrics: a placebo- and standard-controlled clinical investigation. Fournal of Clinical Psychopharmacoly, 2, 122-126.

Pollock, B. G. et al. (2002). Comparison of citalopram, perphenazine, and placebo for the acute treatment of psychosis and behavioral disturbances in hospitalized, demented patients. American fournal of Psychiatry, 159, 460-465.

Porsteinsson, A. P. et al. (2014). Effect of citalopram on agitation in Alzheimer's disease: the cited randomized clinical trial. $\mathcal{F} A M A, 311,682-691$.

Rabinowitz, J., Katz, I., De Deyn, P. P., Greenspan, A. and Brodaty, H. (2007). Treating behavioral and psychological symptoms in patients with psychosis of Alzheimer's disease using risperidone. International Psychogeriatrics, 19, 227-240.

Raivio, M. M., Laurila, J. V., Strandberg, T. E., Tilvis, R. S. and Pitkala, K. H. (2007). Neither atypical nor conventional antipsychotics increase mortality or hospital admissions among elderly patients with dementia: a two-year prospective study. American fournal of Geriatric Psychiatry, 15, 416-424.

Ramos, B. P. and Arnsten, A. F. (2007). Adrenergic pharmacology and cognition: focus on the prefrontal cortex. Pharmacology E Therapeutics, 113, 523-536.

Raskind, M. A., Peskind, E. R., Halter, J. B. and Jimerson, D. C. (1984). Norepinephrine and MHPG levels in CSF and plasma in Alzheimer's disease. Arcives of General Psychiatry, 41, 343-346.

Raskind, M. A. et al. (2007). A parallel group placebo controlled study of prazosin for trauma nightmares and sleep disturbance in combat veterans with post-traumatic stress disorder. Biological Psychiatry, 61, 928-934.

Raskind, M. A. et al. (2013). A trial of prazosin for combat trauma PTSD with nightmares in active-duty soldiers returned from Iraq and Afghanistan. American fournal of Psychiatry, 170, 1003-1010.

Roth, B. L., Sheffler, D. J. and Kroeze, W. K. (2004). Magic shotguns versus magic bullets: selectively non-selective drugs for mood disorders and schizophrenia. Nature Reviews Drug Discovery, 3, 353-359.

Russo-Neustadt, A., Zomorodian, T. J. and Cotman, C. W. (1998). Preserved cerebellar tyrosine hydroxylase-immunoreactive neuronal fibers in a behaviorally aggressive subgroup of Alzheimer's disease patients. Neuroscience, 87, 55-61.

Schneider, L. S., Dagerman, K. S. and Insel, P. (2005). Risk of death with atypical antipsychotic drug treatment for dementia: meta-analysis of randomized placebo-controlled trials. $\mathcal{F} A M A, 294,1934-1943$.

Simoni-Wastila, L., Ryder, P. T., Qian, J., Zuckerman, I. H., Shaffer, T. and Zhao, L. (2009). Association of antipsychotic use with hospital events and mortality among medicare beneficiaries residing in long-term care facilities. American fournal of Geriatric Psychiatry, 17, 417-427.

Street, J. S. et al. (2000). Olanzapine treatment of psychotic and behavioral symptoms in patients with Alzheimer's disease in nursing care facilities: a double-blind, randomized, placebo-controlled trial. The HGEU Study Group. Archives of General Psychiatry, 57, 968-976. 
Streim, J. E. et al. (2008). A randomized, double-blind, placebo-controlled study of aripiprazole for the treatment of psychosis in nursing home patients with Alzheimer's disease. American fournal of Geriatric Psychiatry, 16, 537-550.

Szot, P., White, S. S., Greenup, J. L., Leverenz, J. B., Peskind, E. R. and Raskind, M. A. (2006).

Compensatory changes in the noradrenergic nervous system in the locus ceruleus and hippocampus of postmortem subjects with Alzheimer's disease and dementia with Lewy bodies. Fournal of Neuroscience, 26, 467-478.

Szot, P., White, S. S., Greenup, J. L., Leverenz, J. B., Peskind, E. R. and Raskind, M. A. (2007). Changes in adrenoreceptors in the prefrontal cortex of subjects with dementia: evidence of compensatory changes. Neuroscience, 146, 471-480.

Tariot, P. N. et al. (2006). Quetiapine treatment of psychosis associated with dementia: a double-blind, randomized, placebo-controlled clinical trial. American fournal of Geriatric Psychiatry, 14, 767-776.

Tomlinson, B. E., Irving, D. and Blessed, G. (1981). Cell loss in the locus coeruleus in senile dementia of Alzheimer type. Fournal of the Neurological Sciences, 49, 419-428.

Wang, L. Y. et al. (2009). Prazosin for the treatment of behavioral symptoms in patients with Alzheimer's disease with agitation and aggression. American fournal of Geriatric Psychiatry, 17, 744-751.

West, C. H., Ritchie, J. C., Boss-Williams, K. A. and Weiss, J. M. (2009). Antidepressant drugs with differing pharmacological actions decrease activity of locus coeruleus neurons. International fournal of Neuropsychopharmacology, 12, 627-641.

Zhong, K. X., Tariot, P. N., Mintzer, J., Minkwitz, M. C. and Devine, N. A. (2007). Quetiapine to treat agitation in dementia: a randomized, double-blind, placebo-controlled study. Current Alzheimer Research, 4, 81-93. 